\title{
Effect of ferroelectric layers on the magnetocapacitance properties of superlattices-based oxide multiferroics
}

\author{
M.P. Singh ${ }^{1}$, W. Prellier*,1, L. Mechin ${ }^{2}$ and B. Raveau ${ }^{1}$. \\ ${ }^{1}$ Laboratoire CRISMAT, CNRS UMR 6508, ENSICAEN, \\ 6 Bd du Maréchal Juin, F-14050 Caen Cedex, FRANCE. \\ ${ }^{2}$ Laboratoire GREYC, CNRS UMR 6072, ENSICAEN and University of Caen, 6 Bd \\ du Maréchal Juin, F-14050 Caen Cedex, FRANCE.
}

(November 21, 2018)

\begin{abstract}
A series of superlattices composed of ferromagnetic $\mathrm{La}_{0.7} \mathrm{Ca}_{0.3} \mathrm{MnO}_{3}$ (LCMO) and ferroelectric/paraelectric $\mathrm{Ba}_{1-x} \mathrm{Sr}_{x} \mathrm{TiO}_{3} \quad(0 \leq \mathrm{x} \leq 1)$ were deposited on $\mathrm{SrTiO}_{3}$ substrates using the pulsed laser deposition. Films of epitaxial nature comprised of spherical mounds having uniform size are obtained. Magnetotransport properties of the films reveal a ferromagnetic Curie temperature in the range of 145-158 $\mathrm{K}$ and negative magnetoresistance as high as $30 \%$, depending on the type of ferroelectric layers employed for their growth (i.e. ' $x$ ' value). Ferroelectricity at temperatures ranging from $55 \mathrm{~K}$ to $105 \mathrm{~K}$ is also observed, depending on the barium content. More importantly, the multiferroic nature of the film is determined by the appearance of negative magnetocapacitance, which was found to be maximum around the ferroelectric transition temperature (3\% per tesla). These results are understood based on the role of the ferroelectric/paraelectric layers and strains in inducing the multiferroism.
\end{abstract}

*prellier@ensicaen.fr 
After a long gap, interest has been renewed in multiferroic materials, where ferromagnetism and ferroelectricity coexist ${ }^{1}$ and possess multiferroic coupling, i.e., magnetic domains can be switched by applied electric field and likewise electric domains are switched by applied magnetic field. As a consequence, the latter are potential candidates for various novel devices, e.g., multiple state memory elements and electric field controlled ferromagnetic devices. There are two open basic issues in this field of research: first, what is the origin of multiferroism and second, how to enhance them for suitable applications? Since very few multiferroic materials exist in nature or are synthesized in laboratory ${ }^{2-5}$, the scarcity of such materials is a big stumbling block to address the aforementioned issues ${ }^{6}$. It is therefore timing and warranting to design novel multiferroics and characterize them for their essential properties.

To design a new (artificial) multiferroic, usually three distinct approaches can be adopted, which can be described as follows. The first one deals with the doping of suitable elements e.g., ferromagnetic element(s) in ferroelectric host and vice versa. The second is concerning the designing of composites with the ferroelectric and magnetic hosts. The third consists in designing superlattices composed of either bi-components (e.g., one is ferroelectric and other is ferromagnetic layer, or a nanocomposites ${ }^{5}$ ) or of multi-components (i.e., with three or more distinct compound layers) where one will observe the breaking symmetry by which it may be possible to induce ferroelectricity (FE) and/or ferromagnetism (FM). In building the superlattices for multiferroics, the total structure should be an insulator, but FM materials are often conducting. Thus, one has to chose the FM layers in such a way that it should be insulating in nature. To show the multiferroism in materials, various physical characteristics can be utilized, such as magnetocapacitance effect ${ }^{4-6}$. Furthermore, properties of the superlattices basically depend on the various structural details (e.g., thickness, microstructure, composition, strain, etc.) of the individual components and the interfaces among them. Moreover, the lattice mismatch between the individual type of layer materials as well as the one with the substrate, play important roles in determining the interfacial structure, which in turn governs their electronic and magnetic properties. Thus, it is necessary to study the 
properties of superlattices composed of ferroelectric and ferromagnetic components.

In previous works, we have studied superlattices composed of $\operatorname{Pr}_{0.85} \mathrm{Ca}_{0.15} \mathrm{MnO}_{3} / \mathrm{Ba}_{0.6} \mathrm{Sr}_{0.4} \mathrm{TiO}_{3}{ }^{7-9}$ and $\mathrm{La}_{0.7} \mathrm{Ca}_{0.3} \mathrm{MnO}_{3} / \mathrm{BaTiO}_{3}{ }^{10}$ which behave as multiferroics. In addition, it has been shown that their magnetoelectric properties vary with the FE/FM layer thickness ${ }^{7-10}$. In this paper, we attempt to understand the role of ferroelectric/paraelectric layers on multiferroism. To achieve this goal, we have grown a series of superlattices, composed of the $\mathrm{Ba}_{1-x} \mathrm{Sr}_{x} \mathrm{TiO}_{3}$ (BSTO), where $x=0,0.2,0.6,0.8$ and 1, and $\mathrm{La}_{0.7} \mathrm{Ca}_{0.3} \mathrm{MnO}_{3}$ (LCMO), by utilizing the pulsed laser deposition (PLD) process and characterized them by various techniques for their structural and physical properties.

The growth of the superlattices were carried out at $720^{\circ} \mathrm{C}$ in a flowing 100 mTorr $\mathrm{O}_{2}$ ambient on (001)-oriented $\mathrm{SrTiO}_{3}(\mathrm{STO})^{7}$. Based on the previous work, showing the importance of the thickness of the layers into the magnetoelectric properties of the LCMO-BTO superlattices ${ }^{10}$, we have limited our investigation to superlattices corresponding to a total periodicity of 20 unit cells (u.c.). A peculiar attention was paid for the LCMO layers, the latter were limited to the thickness of 5 u.c. in order to get FM insulating behaviors ${ }^{11}$ and BSTO layers was fixed to 15 u.c. Finally, this structure was repeated 25 times in order to get a total thickness of the order of $200 \mathrm{~nm}$.

Crystallinity of the films was examined by X-ray diffraction (XRD). Morphological study of the films was carried out by atomic force microscopy (AFM). Magnetization (M) of the films was measured as a function of temperature $(\mathrm{T})$ and applied magnetic field $(\mathrm{H})$ using a superconducting quantum interference device magnetometer (SQUID). DC electrical properties of films were measured in four point probe configuration. To measure the electrical properties of the films in current-perpendicular-to-the-plane (CPP) geometry, a $\mathrm{LaNiO}_{3}$ electrode was fabricated through a shadow mask ${ }^{8}$. Dielectric properties of the films were investigated by employing a LCR meter and placing the samples in a PPMS system.

The detail XRD study shows the presence of satellite peaks along with the main diffraction peaks leading to the chemical modulation of the order of $8.3 \mathrm{~nm}$, which is very close to $15 \mathrm{a}_{P}$ of $\mathrm{BSTO}+5 \mathrm{a}_{P}$ of LCMO (not shown), where $\mathrm{a}_{P}$ is the bulk lattice parameter 
of the individual perovskite cells. The presence of higher order satellite peaks adjacent to the main reflection peaks, arising from chemical modulation of multilayer structure, indicates that the films were indeed grown coherently. The $\Phi$-scan recorded around the 103 reflection ${ }^{12}$ exhibits four peaks separated by $90^{\circ}$ from each other (not shown), indicating a 4-fold symmetry as expected for the perovskite structures of LCMO and BSTO, confirming that the superlattices indeed grow epitaxially "cube-on-cube". Fig 1a shows the out-ofplane lattice parameter of the various superlattices, as extracted from the XRD data, as a function of $\mathrm{Ba}$ concentration. For comparison, we have also plotted the lattice parameter of bulk BSTO and the theoretical lattice parameter of the superlattices, extracted from the formula $\left[15 \mathrm{a}_{P}\right.$ of $(\mathrm{BSTO})+5 \mathrm{a}_{P}$ of $\left.(\mathrm{LCMO})\right] / 20$, where $\mathrm{a}_{P}$ is the bulk lattice parameter of the individual perovskite cells. From Fig. 1a, it is evident that with decreasing the Ba concentration, the out-of-plane lattice parameter of superlattice approaches its theoretical value. Fig.1b shows a typical 2D-AFM micrographs of LCMO/BTO film and it reveals that the films are comprised of uniform spherical size mounds having an average dimension of $65 \mathrm{~nm}$. Furthermore, morphological parameters, such as the root mean square roughness of the superlattices, were extracted from the $2 \times 2 \mu \mathrm{m}^{2}$ AFM micrographs and found to be of the order of $0.4 \mathrm{~nm}$, i.e., close to the perovskite unit that indicates a very smooth surface.

The $(M-H)$ curve exhibits a well-defined coercivity confirming the ferromagnetic nature of the film. A typical magnetization vs. magnetic field $(M-H)$ loop for $\mathrm{LCMO} / \mathrm{Ba}_{0.6} \mathrm{Sr}_{0.4} \mathrm{TiO}_{3}$ film, recorded at $10 \mathrm{~K}$, is shown in Fig 2a. Furthermore, the temperature-dependence of the magnetization $M(T)$ recorded under 3000 Oe applied magnetic field (inset of Fig 2a) shows the FM Curie temperature $\left(\mathrm{FM}-\mathrm{T}_{C}\right)$ around $158 \mathrm{~K}$. This value is much lower than the observed bulk LCMO $(\sim 250 \mathrm{~K})$ and it is mostly arising due to the substrate-induced strain and the thickness of the layer $(5 \text { u.c. })^{11}$. Thus, the as-grown

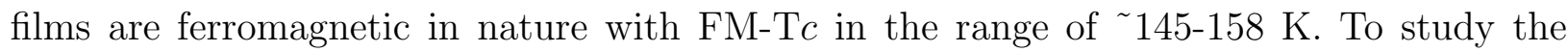
coherent transport through these structures and to examine the effect of the ferroelectric layers on magnetoelectric properties of the superlattices, the resistance $(\mathrm{R})$ was measured under $0 \mathrm{~T}$ and $7 \mathrm{~T}$ magnetic field and the magnetoresistance $(M R)$ is defined as $M R$ 
$(\%)=100 \times[R(H)-R(0)] / R(0)$, where $R(H)$ and $R(0)$ are the resistance values measured with and without magnetic field, respectively. It is well known fact that the intrinsic $\mathrm{MR}$ in manganites is maximum near the $\mathrm{FM}-\mathrm{T}_{C}$ and therefore we have compared here the maximum MR value observed in the various superlattices. Our study shows MR in the superlattices strongly depends on the nature of the ferroelectric layer employed for designing the multiferroic layers (Fig 2b). For example, the superlattice composed of LCMO/BTO reveals a $\mathrm{MR} \sim-30 \%$, whereas a sharp decrease in the MR is observed by substituting a small amount of $\mathrm{Sr}$ in the BTO layer, e.g., the $\mathrm{LCMO} / \mathrm{Ba}_{0.6} \mathrm{Sr}_{0.4} \mathrm{TiO}_{3}$ superlattice exhibits $\mathrm{MR}^{\sim}-12 \%$.

To understand the interaction between ferroelectricity and magnetism, capacitance $(C)$ measurements were performed versus temperature, at $1 \mathrm{KHz}$, under 0 and $5 \mathrm{~T}$. The $C(T)$ curves of these superlattices in $0 \mathrm{~T}$ (Fig 3) exhibit a peak, suggesting ferroelectricity, whatever the Sr content up to $x=0.40$. This observation is in agreement with the fact that the corresponding bulk titanates $\mathrm{Ba}_{0.8} \mathrm{Sr}_{0.2} \mathrm{TiO}_{3}$ and $\mathrm{Ba}_{0.6} \mathrm{Sr}_{0.4} \mathrm{TiO}_{3}$ were also found to be ferroelectric with the transition temperature of $300 \mathrm{~K}$ and $250 \mathrm{~K}$, respectively ${ }^{13,14}$. However, in the present superlattices the transition temperatures are shifted towards much lower values: one observes a transition temperature at ${ }^{\sim} 105 \mathrm{~K}$ for the $\mathrm{LCMO} / \mathrm{Ba}_{0.8} \mathrm{Sr}_{0.2} \mathrm{TiO}_{3}$ superlattice ( Fig 3a) and at ${ }^{\sim} 55 \mathrm{~K}$ for the $\mathrm{LCMO} / \mathrm{Ba}_{0.6} \mathrm{Sr}_{0.4} \mathrm{TiO}_{3}$ (Fig 3b). Hence, the shift of the transition temperature with Sr content supports strongly the presence of ferroelectricity in these films. This view point is furthermore supported by the fact that the $\mathrm{LCMO} / \mathrm{SrTiO}$ superlattice (Fig 3c) does not show any peak of the capacitance in $\mathrm{C}(\mathrm{T})$ curve, in agreement with the absence of ferroelectricity in $\mathrm{SrTiO}_{3}$. The huge deviation of the ferroelectric transition temperature in these superlattices with respect to the bulk materials, can easily be understood by considering nature of ferroelectric layers which are thin ${ }^{15}$ and consequently submitted to large strains from the LCMO layers and indirectly also from the substrate ${ }^{16}$. One must indeed bear in mind that the ferroelectricity in the perovskite structure depends on the nature of the distortion of the $\mathrm{TiO}_{6}$ octahedra and the rattling of $\mathrm{Ti}^{4+}$ inside the octahedra. As a consequence, the ferroelectric transition temperature decrease drastically 
as the size of the A-site cation decreases, e.g., tetragonal- $\mathrm{BaTiO}_{3}$ is ferroelectric, whereas cubic-SrTiO 3 is paraelectric, and $\mathrm{CaTiO}_{3}$ which exhibits another kind of distortion ${ }^{14}$, is also paraelectric. Thus, the $\mathrm{La}^{2+} / \mathrm{Ca}^{2+}$ cations located at the border of the BSTO perovskite layers, may induce particular distortion in the BSTO layer, which represents $~ 5 \%$ of the $A$-sites of the ferroelectric layers, and may decrease drastically the ferroelectric transition temperature.

The second important feature deals with the magnetocapacitance (MC), defined as $M C$ $(\%)=100 \times[C(H)-C(0)] / C(0)$, where $C(H)$ and $C(0)$ are the capacitance measured with and without magnetic field, respectively. Fig 3(a)-(c) show the $C(T)$ curves for various superlattices measured at different magnetic fields. One indeed observes that these superlattices exhibit a negative $M C$ (Fig 3a-b). More importantly, the $M C$ value is found to be maximum near the ferroelectric transition temperature, exactly as the intrinsic magnetoresistance was found to be maximum near $\mathrm{FM}_{C} \mathrm{~T}_{C}$ in the colossal- $M R$ manganites. In contrast, above the $F E$ transition temperature the $M C$ disappears, as shown for instance at the FM-T ${ }_{C}$ of these superlattices $(\sim 150 \mathrm{~K})$. These observations demonstrate that the presence of ferroelectricity is absolutely necessary for the appearance of magnetocapacitance. Importantly, $M C$ is very sensitive to the barium content ( Fig 3d), i.e., to the nature of the $F E$ layers, realizing the highest value for the LCMO/BTO superlattice (i.e. $x=0$ ), decreasing abruptly for $\mathrm{LCMO} / \mathrm{Ba}_{0.8} \mathrm{Sr}_{0.2} \mathrm{TiO}_{3}(x=0.2)$, and so that no magnetocapacitance can be observed for the $\mathrm{LCMO} \mathrm{SrTiO}_{3}$ superlattice $(x=1)$, which does not exhibit any ferroelectricity. On the other hand, the magnetic properties of the manganites are governed by their structural distortion ${ }^{17}$. In other words, by varying the $\mathrm{Mn}^{3+}{ }_{-} \mathrm{O}-\mathrm{Mn}^{4+}$ bond angle, it is possible to tune the ferromagnetism in manganites. The strain in the film plays a major role in determining the $\mathrm{Mn}^{3+}-\mathrm{O}-\mathrm{Mn}^{4+}$ structure ${ }^{18}$. Hence, this study shows that in a superlattice built up of ferromagnetic and ferroelectric layers, magnetocapacitance can only be obtained in the temperature range where both the ferromagnetism and ferroelectricity coexist. More importantly, for designing a multiferroic lattice, it will be crucial to find an optimum stress/strain in the film in order to induce the required interaction for generating 
multiferroism. Thus, the presence of multiferroism in the superlattices will be dependent on the details of FE and FM layers employed for designing them.

To summarize, we have successfully grown LCMO/BSTO superlattices on (001)-oriented $\mathrm{SrTiO}_{3}$ by PLD. The ferroelectric transition temperature is very much dependent on Ba composition. The physical measurements on these superlattices show that the multiferroic properties of the superlattices depend on the type of ferroelectric/paraelectric layers used. Finally, a dependence of the ferroelectricity on the multiferroism has been clearly demonstrated. Furthermore, this study provides a way to design new multiferroics and understand their behaviors which will lead to the realization of the devices based upon them.

We thank Dr. Ch. Simon for helpful discussions. This work has been carried out in the frame of the Work Package "New Architectures for Passive Electronics" of the European Network of Excellence "Functionalized Advanced Materials Engineering of Hybrids and Ceramics" FAME (FP6-500159-1) supported by the European Community, and by Centre National de la Recherche Scientifique. 


\section{REFERENCES}

${ }^{1}$ H. Schmid, Ferroelectrics 162, 317 (1994).

${ }^{2}$ J. Wang, J.B. Neaton, H. Zheng, V. Nagarajan, S.B. Ogale, B. Liu, D. Viehland, V. Vaithyanathan, D.G. Schlom, U.V. Waghmare, N.A. Spaldin, K.M. Rabe, M. Wutting, and R. Ramesh, Science 299, 1719 (2003).

${ }^{3}$ B. Lorenz, F. Yen, M.M. Gospodivno, and C.W. Chu, Phys. Rev. B 71, 014438 (2005).

${ }^{4}$ T. Kimura, S. Kawamoto, I. Yamada, M. Azuma, M. Takano, and Y. Tokura, Phys. Rev. B 67, 180401(R) (2003);

${ }^{5}$ H. Zheng, J. Wang, S.E. Lofland, Z. Ma, L.M. Ardabili, T. Zhao, L.S. Riba, S.R. Shinde, S.B. Ogale, F. Bai, D. Viehland, Y. Jia, D.G. Schlom, M. Wutting, A. Roytburd, and R. Ramesh, Science 303, 661 (2004).

${ }^{6}$ W. Prellier, M.P. Singh, and P. Murugavel, J. Phys.: Condens. Matter 17, R803 (2005).

${ }^{7}$ P. Murugavel, D. Saurel, W. Prellier, Ch. Simon, and B. Raveau, Appl. Phys. Lett. 85, 4424 (2004).

${ }^{8}$ P. Murugavel, P. Padhan, and W. Prellier Appl. Phys. Lett. 85, 4992 (2004).

${ }^{9}$ P. Murugavel, M.P. Singh, W. Prellier, Ch. Simon, B. Mercey, and B. Raveau, J. Appl. Phys. 97, 103914 (2005).

${ }^{10}$ M.P. Singh, W. Prellier, Ch. Simon, and B. Raveau, Appl. Phys. Lett. 87, 022505 (2005).

${ }^{11}$ M.G. Blamire, B.-S. Teo, J.H. Durrel, N.D. Mathur, Z.H. Barber, J.L.M. Driscoll, J.F. Cohen, and J.F. Evetts, J. Magn. Magn. Mater. 167, 200 (1997).

${ }^{12}$ The indexation of the film is based on a perovskite subcell assuming that the film is $c$-axis oriented.

${ }^{13}$ G.A. Smolenski and V.A. Isupov, Sov. J. Techn. Phys. 24, 1375 (1954). 
${ }^{14}$ M.E. Lines and A.M. Glass, Principles and Applications of Ferroelectrics and Related Materials (Oxford Univ. Press, Oxford, 1996).

${ }^{15}$ C.B. Parker, J.P. Maria, and A.I. Kingon, Appl. Phys. Lett. 81, 340 (2002).

${ }^{16}$ S.F. Karmaneko, A.I. Dedyk, A.A. Melkov, R.N. Il'in, V.I. Sakharov, I.T. Serenkov, and J. Ha, J. Phys. Condens. Matter 14, 6823 (2002).

${ }^{17}$ H.Y. Hwang, T.T.M. Palstra, S.W. Cheong, and B. Batloog, Phys. Rev. B. 52, 15046 (1995).

${ }^{18}$ W. Prellier, Ph. Lecoeur and B. Mercey, J. Phys.: Condens. Matter 13, R915 (2001). 


\section{Figure captions:}

Figure 1: (a) : Out-of-plane experimental lattice parameter of the LCMO/BSTO superlattices as a function of Ba content. The lattice parameters of bulk BSTO and the theoretical values of the LCMO/BSTO superlattices are also shown for comparison. (b) 2D: AFM micrograph of the $\mathrm{LCMO} / \mathrm{BaTiO}_{3}$ superlattices.

Figure 2: (a) $(M-H)$ loop of $\mathrm{LCMO} / \mathrm{Ba}_{0.6} \mathrm{Sr}_{0.4} \mathrm{TiO}_{3}$ superlattices measured at $10 \mathrm{~K}$. Inset shows the corresponding $(M-T)$ curve measured under 3000 Oe applied magnetic field. (b) MR of the LCMO/BSTO superlattices as a function of Ba composition. The solid

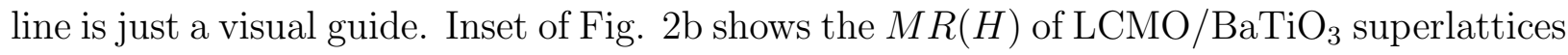
measured at $100 \mathrm{~K}$.

Figure 3. $C(T)$ curves of (a) $\mathrm{LCMO} / \mathrm{Ba}_{0.8} \mathrm{Sr}_{0.2} \mathrm{TiO}_{3}$, (b): $\mathrm{Ba}_{0.6} \mathrm{Sr}_{0.4} \mathrm{TiO}_{3}$, and (c) LCMO/SrTiO 3 superlattices measured under different applied magnetic fields. (d) Negative $M C$ of the LCMO/BSTO superlattice as a function of Ba-composition. The solid line is a visual guide to the eyes. 